\title{
Serendipitous discovery of seven new southern L-dwarfs
}

\author{
T. R. Kendall ${ }^{1}$, N. Mauron ${ }^{2}$, M. Azzopardi ${ }^{3}$, and K. Gigoyan ${ }^{4, \star}$ \\ 1 Centro de Astronomia e Astrofísica da Universidade de Lisboa, Observatório Astronómico de Lisboa, \\ Tapada da Ajuda, 1349-018 Lisboa, Portugal \\ 2 Groupe d'Astrophysique, UMR-5024 CNRS, Case 072, place Bataillon, 34095 Montpellier Cedex 05, France \\ 3 IAM, Observatoire de Marseille, 2 place Le Verrier, 13248 Marseille Cedex 04, France \\ 4378433 Byukaran Astrophysical Observatory \& Isaac Newton Institute of Chile, Armenian Branch, \\ Ashtarak d-ct, Armenia
}

Received 16 December 2002 / Accepted 12 February 2003

\begin{abstract}
We report the discovery of seven hitherto unknown L-dwarfs found as a result of a spectroscopic search for distant AGB stars. Their far-red and near-infrared colours are very similar to known dwarfs of the same spectral type. One new object is among the $\sim 30$ brightest L-dwarfs, with $K_{\mathrm{s}}=12.12$, and is nearby, $\sim 20 \mathrm{pc}$. Using low resolution spectroscopy from the Danish $1.54 \mathrm{~m}$ ESO telescope, spectral types in the range L0.5-L5 are derived for these seven L-dwarfs by direct comparison to L-type standards taken from Kirkpatrick et al. (1999). Distances are determined from existing calibrations, and together with measured proper motions, yield kinematics for the seven new dwarfs consistent with that expected for the solar neighbourhood disk population.
\end{abstract}

Key words. stars: low mass, brown dwarfs - stars: late-type - stars: kinematics - stars: distances - infrared: stars - surveys

\section{Introduction}

Recent near-infrared surveys, most notably 2MASS (Skrutskie et al. 1997) and DENIS (Epchtein 1997) are an invaluable resource for finding and characterising the reddest existing objects. Among galactic objects, one can mention the cool AGB stars, which may be deeply embedded in circumstellar dust, and the L/T dwarf population. The latter such objects, when observed in the field, are likely to have ages of a few Gyr and, with reference to theoretical models (Burrows et al. 1997; Baraffe et al. 1998), to be substellar. As such, ever since the discovery of the first such objects in the solar neighbourhood, GD 165B (Becklin \& Zuckerman 1988) and Gliese 229 B (Nakajima et al. 1995), they have been the subject of intense observational scrutiny. Current and recent studies include Delfosse et al. (1997); Delfosse et al. (1999); Kirkpatrick et al. (1999); Martín et al. (1999); Reid et al. (2000); Fan et al. (2000) and Schneider et al. (2002) and references therein.

It is interesting to note that during a survey devoted to the search for L and T-dwarfs, four dusty AGB carbon stars were discovered at high galactic latitude $\left(|b| \geq 25^{\circ}\right)$, as reported by Liebert et al. (2000). Such luminous AGB stars are invaluable probes of the distant halo (see for example

Send offprint requests to: T. R. Kendall,

e-mail: tkendall@oal.ul.pt

* Based on observations made with the ESO Danish $1.54 \mathrm{~m}$ telescope, La Silla, program 69.B-0186(A).
Totten \& Irwin 1998). In this paper, we report the inverse case: the discovery of seven new L-dwarfs found during a systematic spectroscopic survey of AGB candidates.

Our program to search for high latitude AGB stars is still in progress and will be described fully elsewhere (Mauron et al. 2003, in prep.). Briefly, AGB candidates are selected either from objective prism plates (Gigoyan et al. 2001) or by using a combination of near-infrared and optical colours. The majority of them have $R \sim 13-19$. However, a subset of ten candidates which are invisible on POSS $R$-plates, but relatively bright in the 2MASS $K_{\mathrm{s}}$-band $\left(K_{\mathrm{s}}=11-14\right)$ were immediately selected for further examination using deeper $R$ and $I$-band imaging, to attempt to detect the object in the $R$-band. Subsequently, low resolution $(30 \AA$ ) grism spectroscopy was carried out (see below).

Of the ten objects followed up in this way, seven are shown to be hitherto unknown L-dwarfs. Two were found to be in the Kirkpatrick database (version 6 December 2002) ${ }^{1}$, hereafter K02, which now contains 250 objects classified as spectral type L. These are DENIS-P J0205.4-1159 (Delfosse et al. 1997) and 2MASS J0144353-071614 (Liebert et al. 2003). A further one was found in recent literature by searching the object coordinates in SIMBAD: this is SDSS J042348.57041403.5, listed by Schneider et al. (2002); Geballe et al. (2002), classified T0.

\footnotetext{
${ }^{1}$ http://spider.ipac. caltech.edu/staff/davy/ARCHIVE
} 
Table 1. Basic and derived data for observed targets. The abbreviated name $2 \mathrm{MJhhmm}$ will be used throughout this paper. Previously known objects are referenced in the final column. Coordinates are from 2MASS 2nd incremental release. I magnitudes are taken from UKST I photometry from SuperCosmos. $J H K_{\mathrm{s}}$ magnitudes are from the 2MASS database. Galactic latitudes, derived absolute $J$-magnitudes and distances (where applicable, see text) are given in Cols. 7-9. Spectral types gathered from previous literature are given in parentheses.

\begin{tabular}{lllllllllll}
\hline \hline Name & 2MASS & $I$ & $J$ & $H$ & $K_{\mathrm{s}}$ & $b$ & $M_{J}$ & Sp. & $d /$ pc & Ref.* \\
\hline 2MJ0006 & 2MASSI J0006205-172051 & 18.86 & 15.71 & 14.63 & 14.02 & -75.7 & 12.45 & L2.5 & 45 & - \\
2MJ0013 & 2MASSI J0013578-223520 & 19.42 & 15.75 & 14.57 & 14.01 & -80.4 & 12.89 & L4 & 37 & - \\
2MJ0032 & 2MASSI J0032431-223727 & 18.56 & 15.38 & 14.52 & 13.97 & -83.8 & 12.01 & L1 & 48 & - \\
2MJ0034 & 2MASSI J0034568-070601 & 18.74 & 15.55 & 14.56 & 13.95 & -69.6 & 12.60 & L3 & 39 & - \\
2MJ0125 & 2MASSI J0125369-343505 & 18.30 & 15.48 & 14.47 & 13.86 & -79.5 & 12.31 & L2 & 43 & - \\
2MJ0144 & 2MASSI J0144353-071614 & 18.08 & 14.19 & 13.00 & 12.28 & -66.5 & 13.18 & (L5) & 18 & L02 \\
2MJ0205 & 2MASSI J0205293-115930 & 17.83 & 14.58 & 13.59 & 12.98 & -70.0 & 13.76 & (L7) & 11 & D97 \\
2MJ0423 & 2MASSI J0423486-041403 & 18.40 & 14.45 & 13.44 & 12.94 & -34.5 & - & (T0) & - & G02, S02 \\
2MJ0428 & 2MASSI J0428510-225323 & 16.96 & 13.58 & 12.70 & 12.12 & -40.9 & 11.88 & L0.5 & 22 & - \\
2MJ0443 & 2MASSI J0443058-320209 & 17.81 & 15.27 & 14.34 & 13.87 & -40.0 & 13.18 & L5 & 26 & - \\
\hline
\end{tabular}

* L02: Liebert et al. (2003); D97: Delfosse et al. (1997); G02, S02: Geballe et al. (2002), Schneider et al. (2002).

\section{Observations and data reduction}

The observations were carried out with the $1.54 \mathrm{~m}$ Danish telescope at the European Southern Observatory in La Silla (Chile), from August 29 to Sept. 3, 2002. The weather was good and the seeing varied from 0.9 to $2^{\prime \prime}$.

We used the DFOSC focal reducer which is designed to permit both direct imaging onto a $2048 \times 2048$ CCD through a variety of filters and slit spectroscopy with grisms. For each program source, 2 min exposure images in Bessell $R$ and Gunn $i$ bands were first achieved for source identification. This step is necessary to see whether the source is point-like or extended (and hence discarded) and to see rapidly if the counterpart is also red in $R-I$. This imaging is also useful to note if there is a possible confusion with another close object, which is the case for 2 new objects where a faint galaxy was found to be within a few arcsec of the target (see Figs. 7 and 9). Pre-imaging also permits a rough estimate of the source counts in the $R$-band filter to decide whether low or medium resolution spectroscopy should be attempted.

Spectroscopy was then performed with the source positioned on a $1.5^{\prime \prime}$ slit and DFOSC grisms 8 or 12 , which provide dispersions of $1 \AA$ pix $^{-1}$ and $12 \AA$ pix $^{-1}$ respectively, i.e. 2.5 pixel resolutions of 2.5 and $30 \AA$. Exposure times ranged between $10 \mathrm{~min}$ and $1 \mathrm{hr}$. The intermediate resolution spectra cover $5850-8400 \AA$, while the observed range at low resolution is $5600-10000 \AA$.

Imaging flat-fields in $R$ and $I$-bands were achieved on the telescope dome, as well as spectroscopic dome flats with the slit and the grisms present in the optical beam.

Spectra of the photometric standard white dwarf GD50 were secured with the same $1.5^{\prime \prime}$ slit as for the targets and were used to flux calibrate the final target spectra. These fluxes are of uncertain absolute accuracy, possibly not better than a factor of 2, although for a few objects comparison of two spectra taken on different conditions and on two different dates suggests agreement to within $\sim 20 \%$. Nevertheless, when comparing fluxes from wavelength to wavelength, the quality of the spectra is quite sufficient to allow comparison with calibrated templates and derive spectrophotometric indices (see below).

Data reduction was performed with the MIDAS software installed at Montpellier. After bias subtraction and flat-field correction of the 2-D spectra, a one-dimensional spectrum of the source was extracted and the sky spectrum measured close to the source location was subtracted. Cosmic ray hits were removed manually. No correction for telluric absorption has been performed.

For wavelength calibration of the low resolution spectra, it was found necessary to select and use the few strong and isolated (unblended at low resolution) lines provided by the sky spectrum, together with several of the lines provided by separate He-Ne lamp exposures. Only sky lines were used for the medium-resolution spectrum of $2 \mathrm{MJ} 0144$. The residuals in the dispersion relation were found to be $<1 / 3$ pixel ( $~ 1 / 6$ of a resolution element).

The main defect of the low resolution spectra is the presence of interference fringes for wavelengths beyond $\sim 8500 \AA$. These fringes do not cancel out easily by flat fielding: they are not a spatially stable pattern in CCD spectral frames and vary from exposure to exposure due to mechanical flexure of the instrument. Therefore, the spectra which are displayed here could not be completely corrected. However, our conclusions do not rely heavily on fringe-affected regions.

\section{Results and discussion}

Table 1 gives details of all 10 objects. We include 2MASS photometry for all the objects, and $I$ magnitudes found by searching the SuperCosmos Sky Surveys database (Hambly et al. 2001a-c), which consists of digitised data derived from ESO and UKST Schmidt plates. For the new objects, we draw attention to the high galactic latitude (Col. 7 in Table 1) and include spectral types and distances, for which details of the derivation will be given below.

In Fig. 1, we have plotted our seven new objects (filled squares) and 161 objects with errors in $J-H$ and 


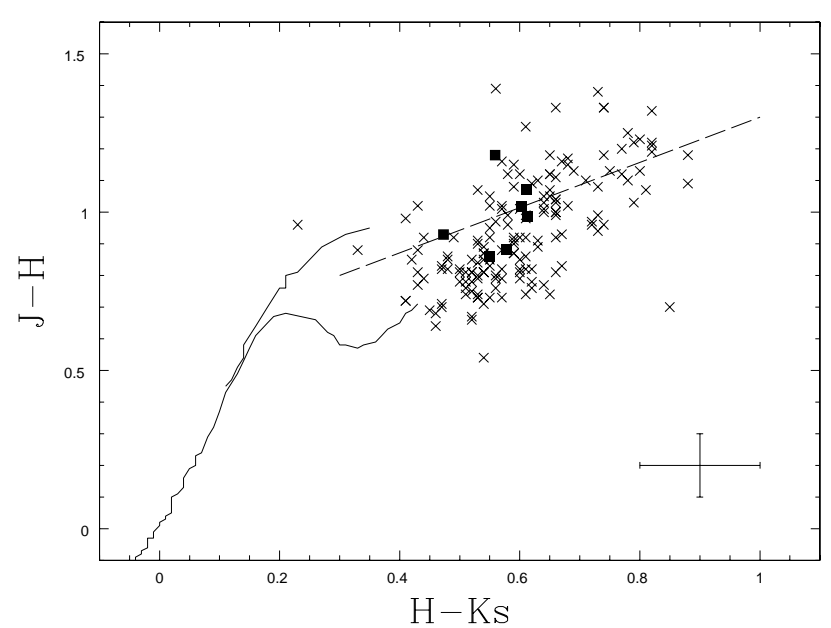

Fig. 1. Near-infrared 2MASS colour-colour diagram for the seven new L-dwarfs (large filled squares) compared to a subset 161 known L-dwarfs from $\mathrm{K} 02$ (crosses) with well-defined $J H K_{\mathrm{s}}$ magnitudes (errors on $J-H$ and $H-K_{\mathrm{s}} \leq 0.1 \mathrm{mag}$, the size shown by the errorbar at lower right). Errors on the 2MASS colours for the new L-dwarfs are similar; $\sim \pm 0.08 \mathrm{mag}$. Also shown are the loci for main sequence (the lower line, near $H-K=0.3$ ) and giant stars, together with an indication of the locus where the majority of cool halo carbon stars lie (near to the dashed line).

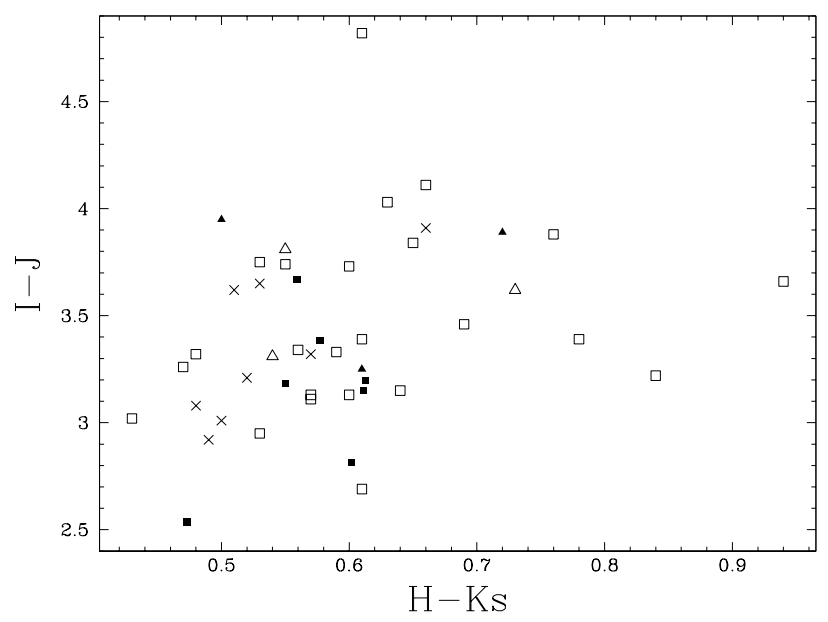

Fig. 2. $I-J, H-K_{\mathrm{s}}$ colour-colour diagram. The 7 filled squares are the seven new L-dwarfs, and the filled triangles are the other three known objects listed in Table 1. Open squares are $24 \mathrm{~L}$-dwarfs from Kirkpatrick et al. (1999), excluding DENIS-P J1228-1547. This object is included in a subsample of objects from Delfosse et al. (1999) which are known L-dwarfs included in K02 (open triangles). Crosses are data from Martín et al. (1999). For consistency, $J H K_{\mathrm{s}}$ magnitudes have been taken from 2MASS in all cases. For our data, $I$ magnitudes have been taken from SuperCosmos, while those of Martín et al. (1999) and Kirkpatrick et al. (1999) are derived from their flux calibrated spectra. Other I magnitudes are from DENIS.

$H-K_{\mathrm{s}} \leq \pm 0.1 \mathrm{mag}$ from $\mathrm{K} 02$ (crosses) in the near-infrared colour-colour diagram. It is clear that the new objects occupy the same region in this diagram as known L-dwarfs. Note that the new object $2 \mathrm{MJ} 0428$ is one of the $\sim 30 \mathrm{~L}$-dwarfs brighter than $K_{\mathrm{s}} \sim 12.2$. In Fig. 2 we compare the new L-dwarfs (filled squares) to a sample from previous literature (for details see

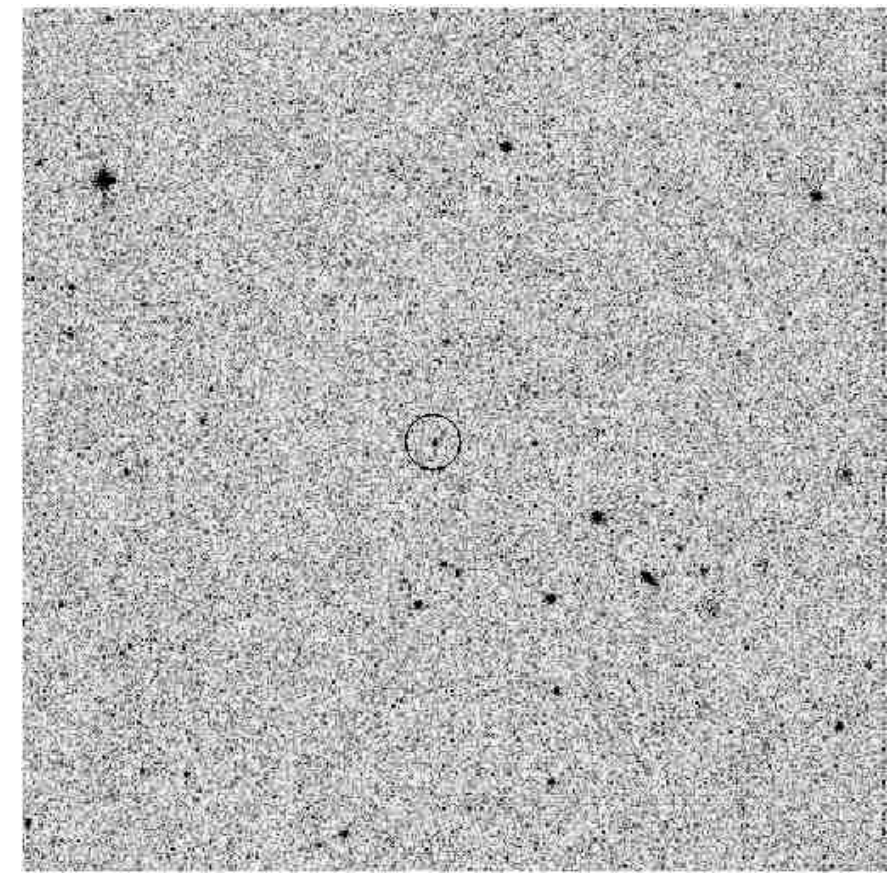

Fig. 3. $I$-band finder chart for $2 \mathrm{MJ} 0006$. All charts presented here are Gunn $i$-band images from the Danish $1.54 \mathrm{~m}$ telescope and are $5^{\prime}$ on a side: $\mathrm{N}$ is up, $\mathrm{E}$ to the left.

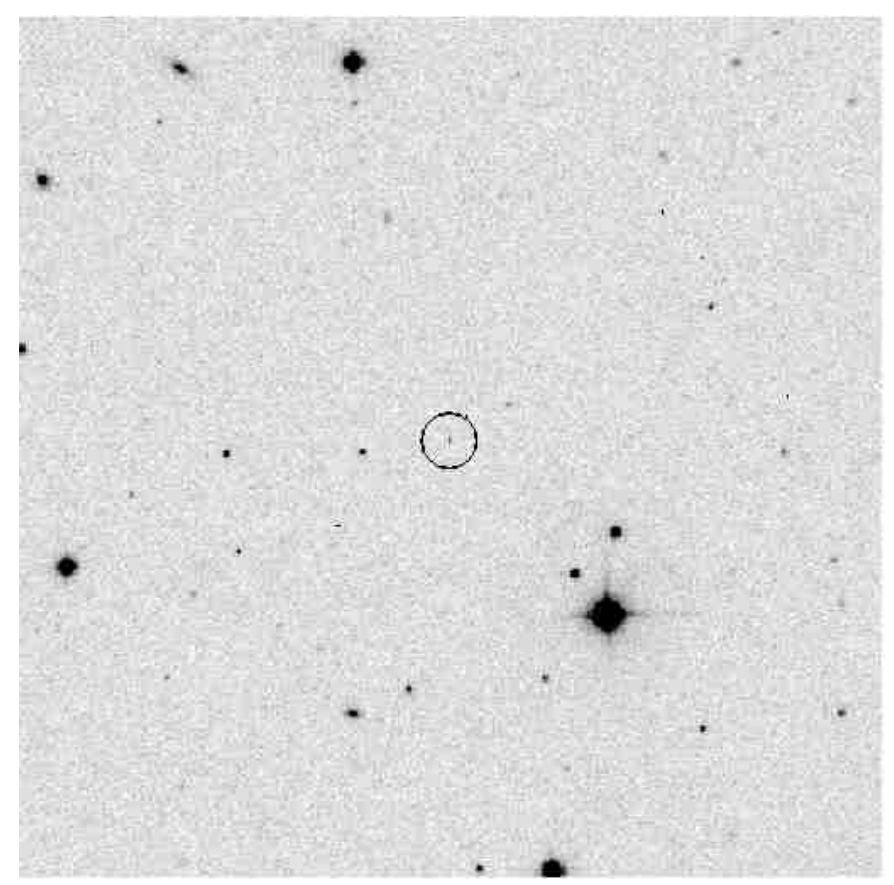

Fig. 4. $I$-band finder chart for $2 \mathrm{MJ} 0013$.

the caption) in the $I-J, H-K_{\mathrm{s}}$ diagram. Note that here we exclude DENIS-P J0205.4-1159 from the sample of Delfosse et al. (1999) (open triangles) and instead plot our data for this object, together with the other 2 previously known objects of Table 1 (filled triangles). $I-J$ has been shown to be a good diagnostic of spectral type, increasing monotonically from late $\mathrm{M}$ to late $\mathrm{T}$, although with more scatter than the $I-z^{*}$ SDSS index (Dahn et al. 2002). Our new objects occupy the region 


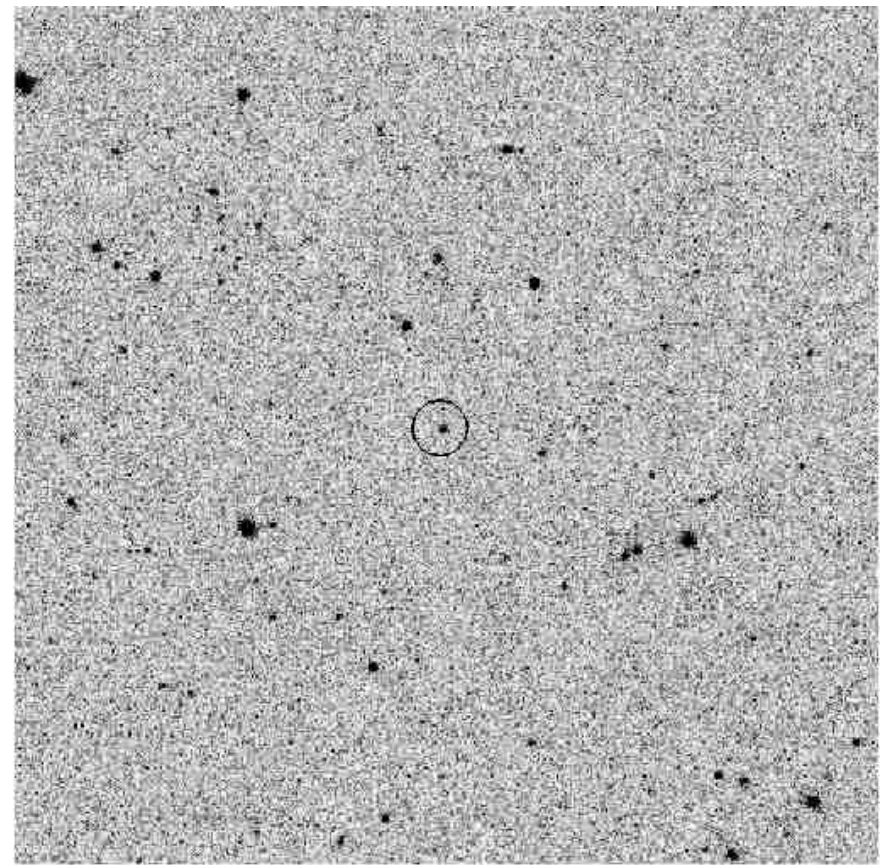

Fig. 5. $I$-band finder chart for $2 \mathrm{MJ} 0032$.

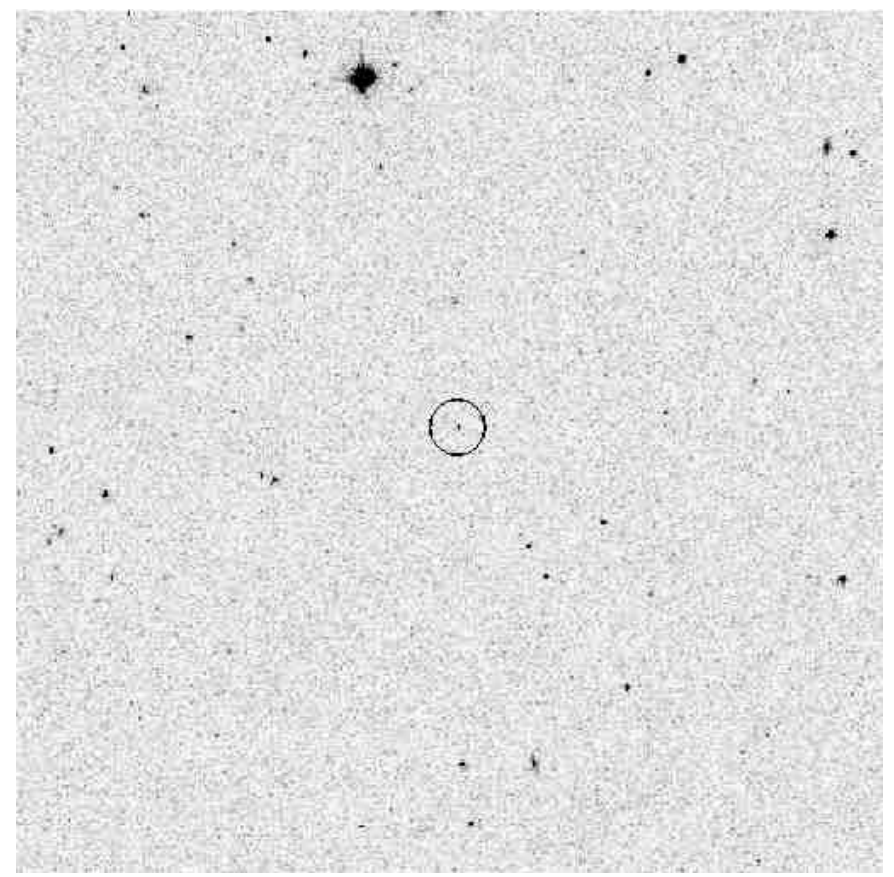

Fig. 6. $I$-band finder chart for $2 \mathrm{MJ} 0034$.

between $\sim 3 \leq I-J \leq \sim 3.7$ which would be expected for objects with spectral types between approximately L0 and L5, although one new object 2MJ0443 (L5) seems anomalously blue for this spectral type. It is possible that the SuperCosmos $I$ photometry has been contaminated by the presence of a faint galaxy (see Fig. 9).

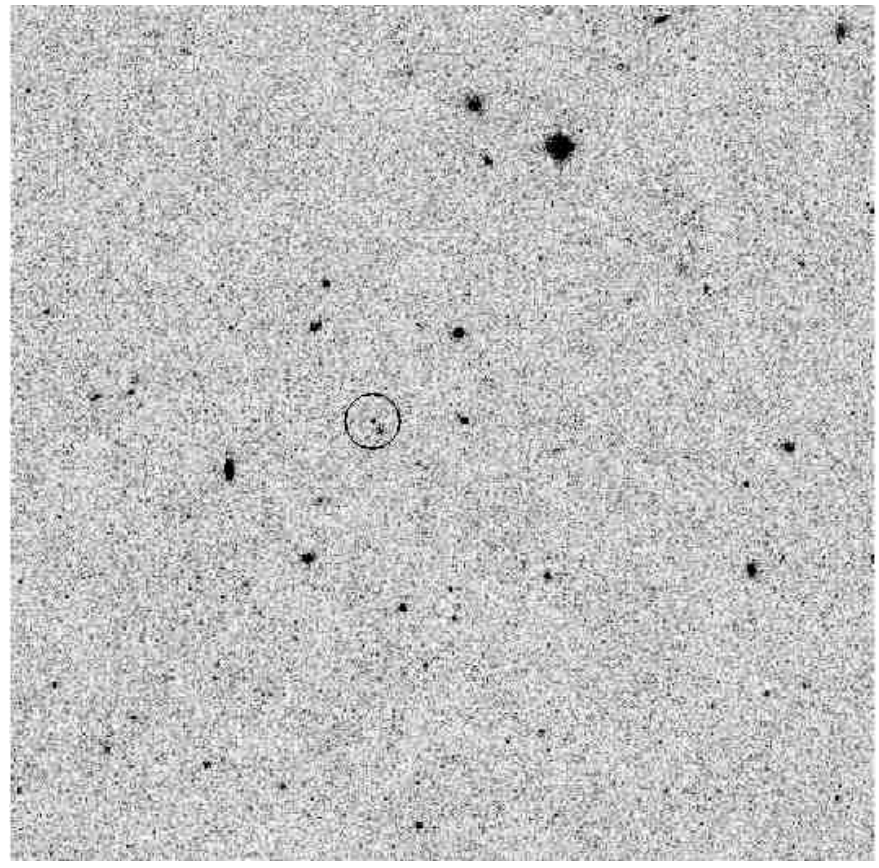

Fig. 7. I-band finder chart for $2 \mathrm{MJ} 0125$. The L-dwarf is the object to the NE of a faint galaxy also within the circle.

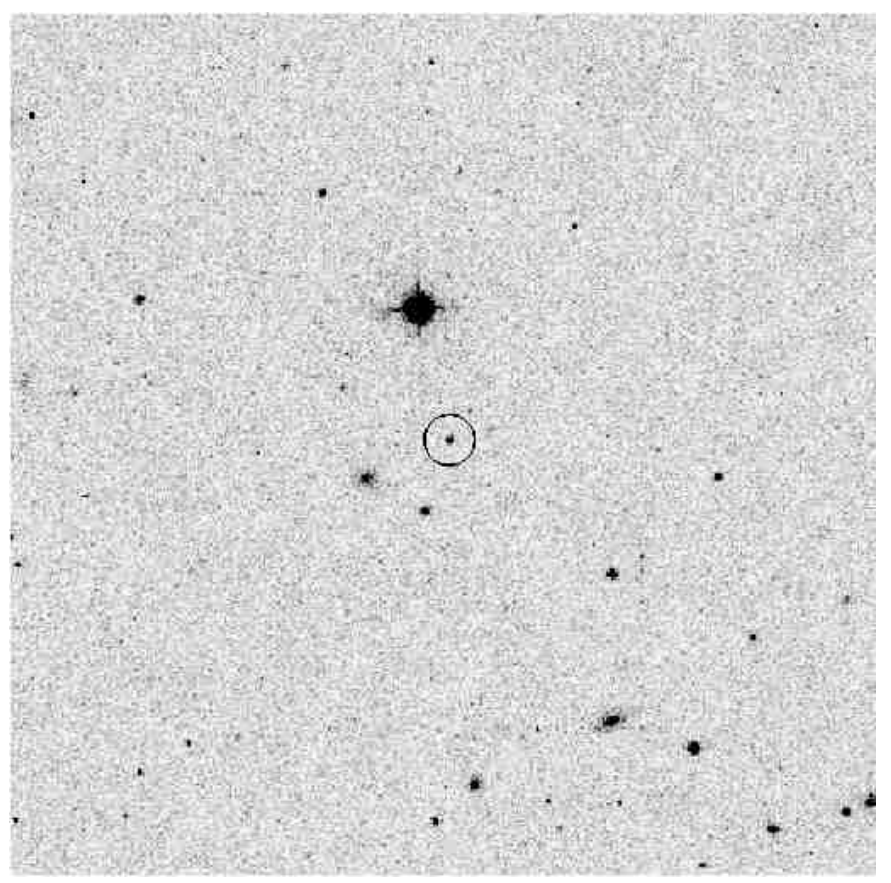

Fig. 8. I-band finder chart for 2MJ0428.

\subsection{Spectral type determinations}

The low resolution of our spectra ( $30 \AA$ ), intended only to allow differentiation between $\mathrm{M}$ and C-type AGB stars, precludes the observation of narrow atomic lines in our L-dwarf spectra. However, as can be seen in Fig. 10, the spectra do exhibit broad $\mathrm{KI}$ and features of $\mathrm{CrH}$ and $\mathrm{FeH}$ which immediately show an L spectral type. The low resolution, and 


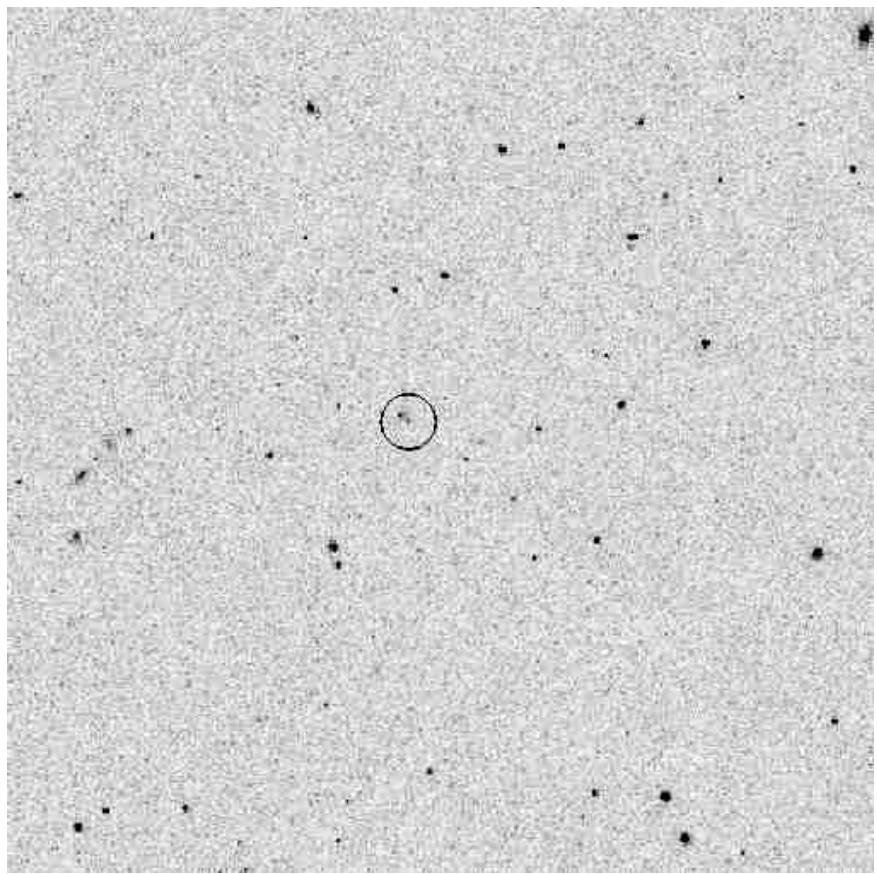

Fig. 9. I-band finder chart for $2 \mathrm{MJ} 0443$. The L-dwarf is the object to the SW of a faint galaxy, also in the circle.

relatively poor signal-to-noise ratio of at least one of the spectra (2MJ0443), preclude the use of spectral typing indices based on ratios of molecular and atomic features. Instead, we have compared our spectra directly with those of L-type standards in the Kirkpatrick et al. (1999) sample. In Fig. 10, the spectra of the seven new L-dwarfs are overplotted with a representative standard star (dotted lines) of the spectral-type indicated in the upper left corner of each panel. This spectral type is the one adopted for the new objects. The spectra of the known T0 and L7 objects SDSS J042348.57-041403.5 and DENIS-P 0205.41159 are also included in this figure for comparison.

When deriving the spectral types, each target spectrum was compared to at least one, and in many cases 3 or 4 standards, including the representative standard we have chosen to plot. The main typing criteria at this resolution were the width of the $\mathrm{K}$ I resonance line, the strength of the $\mathrm{TiO}$ band at $8432 \AA$, and the relative strengths of the $\mathrm{CrH}$ and $\mathrm{FeH}$ bandheads at 8611 and $8692 \AA$. These features are marked in Fig. 10 by vertical bars (together with the broad band near $9300 \AA$ which is due to $\mathrm{H}_{2} \mathrm{O}$, but which includes a significant telluric component). An additional diagnostic of spectral type is the slope of the spectrum near $8000 \AA$, which changes rapidly through very early L-types owing to the disappearance of VO. The spectral typing criteria used here are identical to the major ones used by Kirkpatrick et al. (1999). The excellent agreement between the shape of our new spectra and the chosen standards in Fig. 10 suggests the spectral types for the new objects are accurate to \pm 1 subclass.

Additionally, we have performed experiments using the pseudocontinuum index PC3 of Martín et al. (1999), which increases between early-M and late-L dwarf types but only rather slowly in the early-L regime. The PC3 index compares the slope of the pseudocontinuum over $40 \AA$ ranges centred at $8240 \AA$ and $7560 \AA$ and the relations between it and spectral type for the ranges M2.5 to L1 and L1 to L6 are given by Martín et al. (1999). For the known T0 dwarf SDSS J042348.57-041403.5 (2MJ0423) these calibrations are not appropriate. However, for the other two late L-dwarfs with previously published types (2MJ0144 and 2MJ0205 in Table 1), we find agreement to within 1 subclass from our spectra using the PC3 index.

For one object, 2MASS J014435.3-071614 (2MJ0144, classified L5 (Liebert et al. 2003), a higher resolution (2 A) spectrum was obtained (see Fig. 11), which does not include the hydride bands of $\mathrm{FeH}$ and $\mathrm{CrH}$ or the $8432 \AA$ TiO feature. After rebinning to $8 \AA$ resolution to obtain a sufficient signalto-noise ratio, we obtain a type of L4 (PC3 $=6.54)$.

For the earlier L-type objects, to mitigate against of the very low resolution and moderate signal-to-noise ratio of the spectra, and the fact that the PC3 index is very slowly varying in this range, we increased the width of the 2 PC3 ranges to $100 \AA$. Spectral types of L1 were found for 2MJ0125, 2MJ0006, 2MJ0428 and 2MJ0032 (PC3 = 2.81, 2.69, 2.58 and 2.74 respectively). For $2 \mathrm{MJ} 0034$ we found L2 (PC3 = 3.34), and L3 for the remaining new objects 2MJ0013 and 2MJ0443 (PC3 = 4.39 and 4.74). In most cases the spectral types obtained using PC3 are systematically $\sim 1$ subclass bluer than those found by direct visual comparison with standards. The exception is the lowest signal-to-noise ratio spectrum 2MJ0443, where PC3 suggests L3 instead of L5. These discrepancies suggest that in order to use the PC3 index for these data, it would need to be recalibrated by observing spectral standards with the same instrumental setup. We note that, while the PC3 index is not satisfactory for classification here, it indicates the consistency of the relative spectrophotometric calibration of the data. We adopt the spectral types given by direct comparison with standards for the remainder of this discussion.

\subsection{Distance derivations and proper motions}

We have used the $\mathrm{M}_{J}$-spectral type relations of Hawley et al. (2002) to derive distances, given in Table 1. Two objects, 2MJ0428 (L0.5) and 2MJ0443 (L5) are rather nearby ( 30 pc or less), as close as the $3 \mathrm{~L}$-dwarfs discovered in the proper motion study of Lodieu et al. (2002). We note here that the Hawley et al. (2002) calibrations suggest the L7 object DENISP 0205.4-1159 (2MJ0205) is very close (11 pc). The T0 object SDSS J042348.57-041403.5 (2MJ0423) would also be nearby (12 pc), but we caution that the $\mathrm{M}_{J}$-spectral type relation is non-monotonic in the late- $\mathrm{L}$ to mid- $\mathrm{T}$ regime, as shown by Dahn et al. (2002), so may not be applicable to this object. Importantly, for the case of DENIS-P 0205.4-1159, the distance is known via a trigonometric parallax determination to be $19.8 \mathrm{pc}$, and the discrepancy between this and the $11 \mathrm{pc}$ distance derived from the spectral type is a result of this object being an equal magnitude binary (Dahn et al. 2002). Given that our present sample is magnitude-limited, it is biased towards finding such binaries: one object especially, 2MJ0428, which our results suggest to be rather close $(\sim 22 \mathrm{pc})$ may also be a good candidate to investigate for duplicity. 

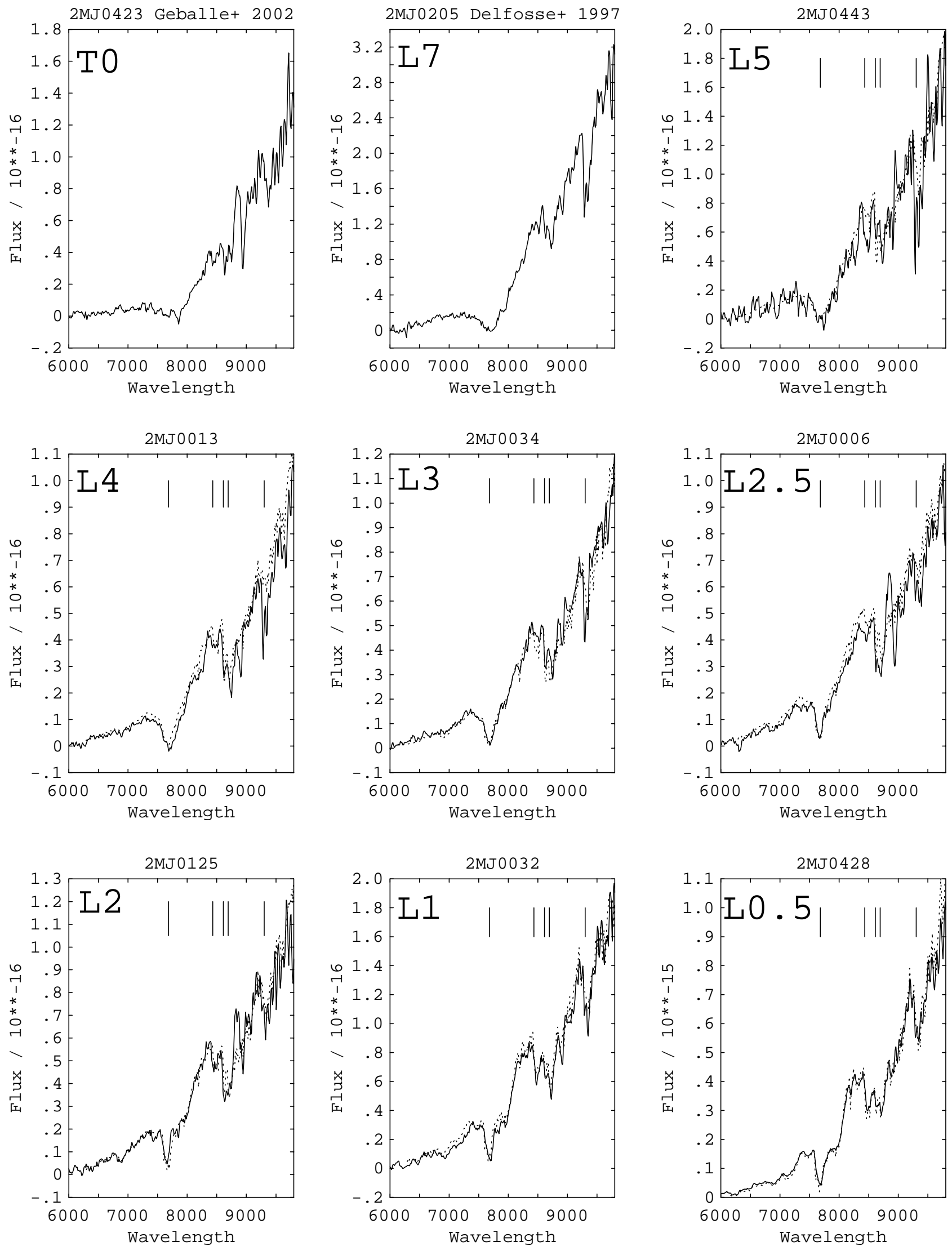

Fig. 10. Low resolution (30 A) spectra of the 7 new objects, plus the 2 known objects SDSS J042348.57-041403.5 (2MJ0423; T0) and DENISP0205.4-1159 (2MJ0205; L7). Spectra are overlain with L-type standards (dotted lines) of the given type from Kirkpatrick et al. (1999). Note 5 features visible at this resolution are marked by vertical bars in order of increasing wavelength. Note K I broadening to later types, the $\mathrm{TiO}$ band (head at $8423 \AA$ ) is visible in all early-L spectra and $\mathrm{CrH}$ and $\mathrm{FeH}$ bandheads at 8611 and $8692 \AA$ respectively. The strong band near $\sim 9300 \AA$ is telluric $\mathrm{H}_{2} \mathrm{O}$. Fringe residuals affect the spectra beyond $8500 \AA$ at the $10-20 \%$ level. Flux units $\operatorname{are} \operatorname{erg~s}^{-1} \mathrm{~cm}^{-2} \AA^{-1}$. 
Table 2. Proper motion determinations for the 7 new objects. For 2 objects, the difference in epoch between SuperCosmos and $2 \mathrm{MASS}$ positional information is too small for the derived motion to be significant. One measurement is clearly anomalously large, and the SuperCosmos position is unreliable because of blending. The penultimate column gives tangential velocity $\left(\mathrm{km} \mathrm{s}^{-1}\right)$ computed using the distances of Table 1 and the proper motions added in quadrature.

\begin{tabular}{llllll}
\hline \hline Name & $\begin{array}{l}\mu_{\alpha} \cos \delta \\
\mathrm{mas} \mathrm{yr}^{-1}\end{array}$ & $\begin{array}{l}\mu_{\delta} \\
\mathrm{mas} \mathrm{yr}^{-1}\end{array}$ & $\begin{array}{l}\Delta_{\text {epoch }} \\
\mathrm{yr}\end{array}$ & $\begin{array}{l}v_{\text {trans }} \\
\mathrm{km} \mathrm{s}^{-1}\end{array}$ & Note \\
\hline 2MJ0006 & 35 & 270 & 1.23 & 59 & $\Delta_{\text {epoch small }}$ \\
2MJ0013 & 100 & 55 & 16.86 & 20 & - \\
2MJ0032 & 90 & 40 & 16.89 & 23 & - \\
2MJ0034 & 200 & 110 & 5.02 & 42 & - \\
2MJ0125 & 180 & 120 & 7.34 & 45 & - \\
2MJ0428 & $\sim 0$ & 170 & 2.88 & 18 & $\Delta_{\text {epoch small }}$ \\
2MJ0443 & 655 & 940 & 2.03 & 143 & anomalous \\
\hline
\end{tabular}

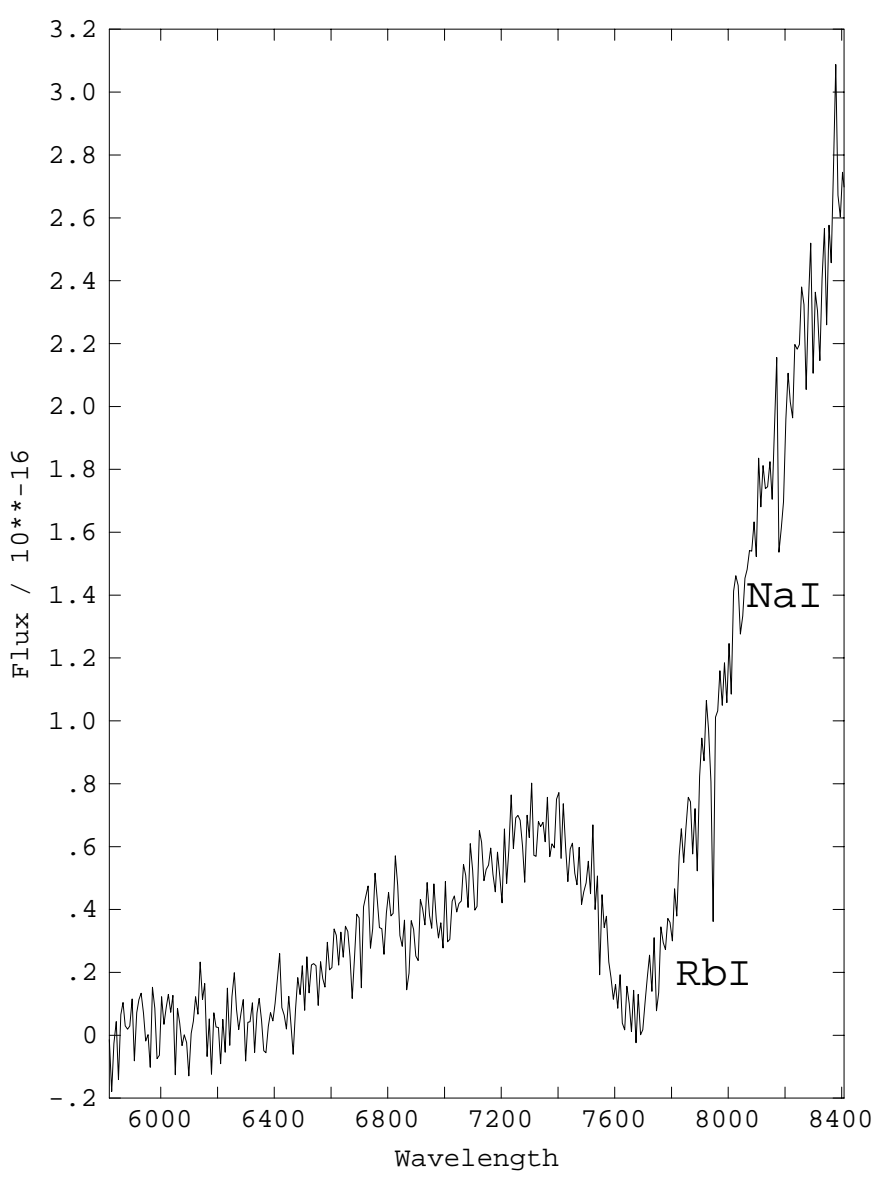

Fig. 11. $2 \AA$ resolution spectrum of the known object 2MASS J014435.3-071614 (2MJ0144) - spectral type L5 - rebinned to $8 \AA$ resolution to clearly show the RbI line at $7948 \AA$ and the Na I doublet (unresolved at this resolution) at $\sim 8190 \AA$. The wavelength range does not include the hydride bands. Note the broad KI. Flux units are $\operatorname{erg~s}^{-1} \mathrm{~cm}^{-2} \AA^{-1}$.

In Table 2, we have computed proper motions using the positional differences in $\alpha$ and $\delta$ between SuperCosmos and 2MASS images taken at different epochs. Column 4 gives the timeline over which the proper motions were derived, and for three objects, we consider that it is too small for the proper motion to be reliable, and for one of these cases (2MJ0443) an anomalously large proper motion is derived. On examination of the SuperCosmos UKST I image for this case, we find that the image, which is resolved into the L-dwarf and a faint galaxy in the Danish I-band image of Fig. 9, is clearly non-stellar and very indistinct, and it is possible that the SuperCosmos position we have used is that of the nearby faint galaxy.

Moreover, we have derived transverse velocities using the spectroscopic distances of Table 1 and the proper motion determinations. For the 2 cases in Table 2 for which $\Delta_{\text {epoch }}$ is perhaps unreliably small, we derive $\sim 60$ and $\sim 20 \mathrm{~km} \mathrm{~s}^{-1}$. However for the remaining four objects, we find $\sim 20,25,40$ and $45 \mathrm{~km} \mathrm{~s}^{-1}$, which are quite consistent with the kinematics of disk stars.

\section{Conclusions}

We have discovered seven new southern L-dwarfs at high galactic latitude serendipitously in a systematic survey for distant halo AGB stars. We have derived spectral types in the range L0.5-L5 for the new objects by direct comparison to L-type spectral standards listed by Kirkpatrick et al. (1999). The seven new L-dwarfs have spectroscopic distances between $~ 20$ and $50 \mathrm{pc}$. Together with the proper motions we have derived by comparing $2 \mathrm{MASS}$ and SuperCosmos positions at different epochs, we have determined transverse velocities consistent with the kinematics of disk stars. Follow-up observations at higher spectral resolution are required to assess spectral types, and hence distances, more accurately by using a larger number of spectral features and indices, and also to address the question of the new objects' masses, and potential substellarity, via observations of the Li I $6708 \AA$ line (Rebolo et al. 1996).

Acknowledgements. TRK acknowledges financial assistance from the European Union Research Training Network "The Formation and Evolution of Young Stellar Clusters" (RTN1-1999-00436). NM thanks the ESO staff, in particular John Pritchard, for help in making the observations. KG thanks the Jumelage 18 "Astrophysique FranceArménie" for support. TRK thanks the referee, J. Davy Kirkpatrick, and Eduardo Martín, for their comments and assistance. Part of this work was carried out during a visit of TRK to the Institute for Astronomy, University of Hawaii, funded by the National Science Foundation (NSF) grant AST-0205862. This publication makes use of data products from the Two Micron All Sky Survey, which is a joint project of the University of Massachusetts and the Infrared 
Processing and Analysis Center/California Institute of Technology, funded by the National Aeronautics and Space Administration and the national Science Foundation. This research has made use of the SIMBAD database, operated at CDS, Strasbourg, France.

\section{References}

Baraffe, I., Chabrier, G., Allard, F., et al. 1998, A\&A, 337, 403 Becklin, E. E., \& Zuckerman, B. 1988, Nature, 336, 656 Burrows, A., Marley, M., Hubbard, W. B., et al. 1997, ApJ, 491, 856 Dahn, C. C., Harris, H. C., Vrba, F. J., et al. 2002, AJ, 124, 1170

Delfosse, X., Tinney, C. G., Forveille, T., et al. 1997, A\&A, 327, L25 Delfosse, X., Tinney, C. G., Forveille, T., et al. 1999, A\&AS, 135, 41 Epchtein, N. 1997, in The Impact of Large-Scale Near-infrared Surveys, ed. F. Garzón, N. Epchtein, A. Omont, B. Burton, \& P. Persi (Dordrecht: Kluwer Academic Publishers), 15

Fan, X., Knapp, G. R., Strauss, M. A., et al. 2000, AJ, 119, 928

Hambly, N. C., MacGillivray, H. T., Read, M. A., et al. 2001a, MNRAS, 326, 1279

Hambly, N. C., Irwin, M. J., \& MacGillivray, H. T. 2001b, MNRAS, 326, 1295

Hambly, N. C., Davenhall, A. C., Irwin, M. J., \& MacGillivray, H. T. 2001c, MNRAS, 326, 1315
Hawley, S. L., Covey, K. R., Knapp, G. R., et al. 2002, AJ, 123, 3409

Geballe, T. R., Knapp, G. R., Leggett, S. K., et al. 2002, ApJ, 564, 466

Gigoyan, K., Mauron, N., Azzopardi, M., Muratorio, G., \& Abrahamyan, H. V. 2001, A\&A, 371, 560

Kirkpatrick, J. D., Reid, I. N., Liebert, J., et al. 1999, ApJ, 519, 802

Liebert, J., Cutri, R. M., Nelson, B., et al. 2000, PASP, 112, 1315

Liebert, J., Kirkpatrick, J. D., Cruz, K. L., et al. 2003, AJ, 125, 343

Lodieu, N., Scholz, R.-D., McCaughrean, M. J. 2002, A\&A, 389, L20

Martín, E. L., Delfosse, X., Basri, G., Goldman, B., \& Zapatero Osorio, M. R. 1999, AJ, 118, 2466

Mauron, N., Gigoyan, K., \& Azzopardi, M. 2003, in preparation

Rebolo, R., Martín, E. L., Basri, G., Marcy, G. W., \& Zapatero-Osorio, M. R. 1996, ApJ, 469, L53

Reid, I. N., Kirkpatrick, J. D., Gizis, J. E., et al. 2000, AJ, 119, 369

Nakajima, T., Oppenheimer, B. R., Kulkarni, S. R., et al. 1995, Nature, 378,463

Schneider, D. P., Knapp, G. R., Hawley, S. L., et al. 2002, AJ, 123, 458

Skrutskie, M. F., Schneider, S. E., Stiening, R., et al. 1997, in The Impact of Large-Scale Near-infrared Sky Surveys, ed. F. Garzón, N. Epchtein, A. Omont, B. Burton, \& P. Persi (Dordrecht: Kluwer Academic Publishers), 25

Totten, E. J., \& Irwin, M. 1998, MNRAS, 294, 1 